\title{
ERRATUM
}

\section{Interaction between light and highly confined hypersound in a silicon photonic nanowire}

Raphaël Van Laer, Bart Kuyken, Dries Van Thourhout and Roel Baets

Nature Photonics 9, 199-203 (2012); published online 16 February 2015; corrected after print 8 May 2015.

In the version of this Article originally published, in the expression for $L_{\mathrm{eff}}$ on page 200 the exponential should have contained a minus sign and the expression should have read $L_{\mathrm{eff}}=(1-\exp (-\alpha L)) / \alpha$. This has been corrected in the online versions. 